\title{
Antaras Aconcagua: un estudio antropológico y acústico
}

\section{«Antaras Aconcagua: an acoustic and anthropological study»}

\author{
Benoit Fabre \\ Université Pierre et Marie Curie, Francia \\ benoit.fabre@upmc.fr
}

José Pérez de Arce

Museo Chileno de Arte Precolombino, Chile

jperezdearce@museoprecolombino.cl

\author{
Patricio de la Cuadra \\ Pontificia Universidad Católica de Chile, Chile \\ patodelac@gmail.com
}

- Resumen - La antara, más que un instrumento musical, fue un importante símbolo cultural, religioso y social al que la cultura Aconcagua destinó sus mejores habilidades artesanales y otorgó un lugar fundamental dentro de su ritualidad. Trazos de su importancia persisten a través de flautas rituales (pifilcas y flautas de chinos), actualmente ejecutados en la región surandina. En este artículo se intenta situar, a través un análisis multidisciplinario que incluye su descripción organológica, antropológica y acústica, las características del instrumento y los ritos que la acompañaron, en un intento de rescatar la memoria de quien fuera un ícono central de la cultura Aconcagua.

Palabras clave: antara, Aconcagua, pifilca, sonido rajado.

Abstract - The Antara, more than a musical instrument, was an important cultural, religious and social symbol. The Aconcagua culture assigned their best artisans to this instrument, which had a crucial positioning in their rituals. Its relevance can still be found in the ritual flutes (pifilcas and "Chino" flutes) that are nowadays executed in the southern Andean region. The following article will discuss the characteristics of the Antaras, and the rituals in which this flute is present, through a multidisciplinary analysis that includes an organological, anthropological and acoustic approach, in an attempt to rescue the memory of a central icon in the Aconcagua culture.

Keywords: antara, Aconcagua, pifilca, Torn sound. 


\section{LA RUTA ANTARA}

La «antara» es una flauta de pan con tubos de geometría interna irregular, llamados tubos complejos, que permiten producir un sonido particular llamado «sonido rajado». La "antara surandina» se encuentra en una extensa zona que abarca desde Potosí, Cochabamba, La Paya, Santa María, San Pedro, Copiapó, Diaguita y Aconcagua, llegando al sur hasta el área mapuche. Hace su aparición con la milenaria tradición Tiwanaku (300 - 1.100 dC.), la cual expande la influencia de su nueva ritualidad, regulada por ciclos de fiestas y ferias, con actividades chamánico-religiosas, hacia Atacama y el noroeste Argentino. Esta «antara» imita el perfil escalerado del «siku» de caña, con sus tubos esculpidos exteriormente y con un asa lateral que adquiere de los instrumentos musicales surandinos. Al pasar a Atacama, y luego al noroeste Argentino, pierde paulatinamente el dibujo exterior de los tubos, quedando con una superficie lisa. También aparece en esa misma región, al parecer en un periodo tardío (Horta 2010), un corpus de representaciones que hemos llamado el «tema del antarista». Se trata de tallados tridimensionales en los objetos de madera del «complejo del rape» que vinculan el uso de la antara surandina con el sacerdote sacrificador, el puma y la llama con la inhalación del cebil. El sacrificador era un tipo especial de chamán, capaz de celebrar la ceremonia del sacrificio humano por decapitación, y tenía el privilegio de tañer la "antara", la cual aparece en ciertas representaciones sustituyendo la cabeza cortada, estableciendo un vínculo de sentido para el instrumento. La víctima que será sacrificada aparece desnuda, en cuclillas, sus manos agarrando las rodillas, con la antara que no es sujeta por las manos (a diferencia de lo que ocurre con báculos y otros objetos representados en otras tabletas) ni apoyada sobre la boca, lo cual parece enfatizar el hecho que la antara se toca sola, fenómeno que ha sido descrito asociado al trance musical. La antara aparece como el elemento más destacado en el tema iconográfico dentro de esta trascendental ceremonia, revelando así la enorme importancia que tuvo este instrumento, lo que concuerda con lo detectado en los contextos funerarios que conocemos.

Posteriormente, esta "antara» llega a territorio diaguita. Su forma externa se comienza a desdibujar; el perfil escalerado en algunos ejemplares se redondea, se hace más orgánico, menos rígido, acorde a los cánones estéticos locales, pero conservan sus características organológicas. Su llegada constituye una propuesta ideológica con nuevas ideas y tecnologías provenientes del norte de Chile y noroeste argentino. Provocan una revolución ideológica, social, política y económica que da origen, en el norte chico, al llamado Complejo Cultural Las Animas y posteriormente a las culturas Copiapó (valles Copiapó y Huasco) y Diaguita (valles Elqui, Limarí, Choapa e Illapel), y en Chile Central, al Complejo Cultural Aconcagua. Este cambio probablemente incluyó la extensión de la llamada «ruta del cebil», un complejo sistema de caravanas de llamas que reparte el cebil desde la ladera oriental de la Sierra de Ancasti, en Catamarca, por una extensa región, junto con la tradición de sacrificadores, cabezas trofeo y decapitados que aparece en los equipos alucinógenos diaguita. 
Así, junto a los cambios en la «antara», se introducen otros menores en el complejo alucinógeno, como la incorporación de tubo de cañón de pluma de cóndor o de hueso de ave marina para la aspiración del polvo alucinógeno (Castillo 109, 121, 154). La permanencia de la parafernalia ritual y de la representación del chamán sacrificador nos indica la importancia que alcanzó esta nueva ritualidad en la región. Este sacrificador alcanzó a ser visto por Mariño de Lobera hacia 1590 en Copiapó, donde invocaba al sol y reverenciaba «unas figuras de ídolos mal formados», y cuyo símbolo de su rango era su hacha de cobre, con la cual decapitaba (Hidalgo 291).

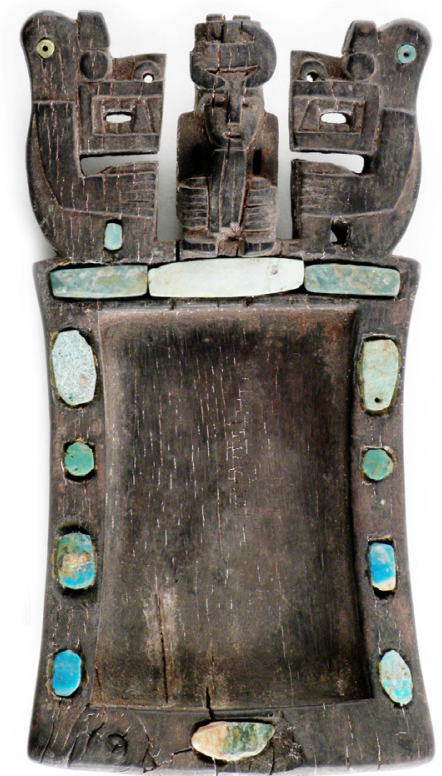

Figura 1. Tableta de rape (Museo San Pedro de Atacama cat. 9160 IIAM 402) con una figura esculpida de un personaje (identificado como víctima por Horta, 2010).

En la región de la cultura Aconcagua, las «antaras» muestran en su aspecto externo el mestizaje con las flautas locales, sin perder sus características organológicas (cuatro tubos complejos en disposición ascendente), alcanzando su mayor grado de estilización y refinamiento. Más al sur, la antara llega hasta la región mapuche, donde sufre una transformación exterior más radical, influenciada por las flautas locales; pierde el escalerado y se hace simétrico, con dos asas y base redonda, pero siempre manteniendo los rasgos organológicos y su uniformidad tipológica.

\section{ARTESANÍA LÍTICA}

La maestría de las «antaras» de piedra Aconcagua sorprende en muchos ejemplares por su extraordinario refinamiento. El grado de perfección y de detalles sugiere que su construcción debe haber sido hecha por artesanos expertos, con conocimientos 
muy refinados de artesanía lítica, acústica y diseño. Su confección requería un enorme tiempo de trabajo y, por lo tanto, una elite, una escuela de artesanos antaristas capaces de traspasar su conocimiento a través del tiempo. El hallazgo de un conjunto de fragmentos de flautas en la localidad de Panquehue, que corresponden a 24 instrumentos diferentes, parece indicar la existencia de este tipo de talleres, situación que difiere de otros sistemas productivos Aconcagua, tales como el de artefactos líticos o de objetos de cerámica, en que las evidencias indican que no hay especialistas, sino que cada comunidad produce los objetos según su necesidad, en forma ocasional. Es decir, la producción de flautas no parece seguir los patrones de producción de otro tipo de objetos en esa cultura. Es muy posible que hubiera una artesanía en madera para los mismos instrumentos, como la hay hoy con las flautas de chinos en la misma región; la madera no se ha conservado en la zona Aconcagua por razones climáticas, pero sí en la zona desértica más al norte, donde tenemos la evidencia de «antaras» de ese material. Es posible, como ocurría hasta hace poco, que cada comunidad tuviera un baile con un especialista constructor de flautas de madera, lo cual les daba una autonomía y un perfil sonoro personal frente a los demás bailes. Las flautas de piedra serían un caso excepcional, instrumentos especialmente diseñados para perdurar, y probablemente cargados de una importancia cultural especial; el caso de Panquehue puede obedecer a esa lógica, a un taller de artesanía especializado en ese tipo muy especial de objetos rituales. Probablemente son objetos hechos para usarse largo tiempo, por más de una generación, y por eso se ha invertido tanta energía en su producción.

En general, el conjunto de "antaras» que conocemos revela una gran comunidad de propósito, que solo se puede conseguir con una red de comunicaciones entre músicos y fabricantes de instrumentos, una tradición oral de gran vigor y un sistema musical asentado en un tiempo y espacio de cierta envergadura. Para las culturas prehispánicas, el material no es solo algo útil para construir artefactos, sino que es también un sustrato cargado de significaciones simbólicas, mágicas, de potencias y de vinculaciones con la naturaleza. Así, como en la cultura Nazca, el sur de Perú alcanza una maestría musical insuperable en la cerámica. Con sus notables «antaras», timbales y flautas, podemos decir que los Aconcagua alcanzaron la maestría musical en piedra. La piedra es un material mucho más difícil de trabajar que la cerámica, aunque su maestría es menos visible. Se despliega a través de detalles constructivos sutiles de tipo organológico, acústico o en destrezas artesanales que pasan desapercibidas para el ojo inexperto, es decir, está al servicio de los iniciados conocedores de sus secretos. Todo esto nos señala que, más que un artesano, probablemente estamos ante una especie de sacerdote - artesano, un sabio poseedor de una llave maestra para el ritual.

El valor dado al material se refleja en que todas las «antaras» Aconcagua conocidas están hechas en un solo tipo de piedra, escogida por su coloración (y tal vez por otros atributos que se nos escapan). Se trata de la piedra combarbalita de 
tonos rojizos, que no se encuentra en la zona, y que debió ser traída desde el norte, a cientos de kilómetros de distancia, desde la región de Illapel (Jackson, com. pers.).

Actualmente, en la región del Aconcagua, la artesanía de flautas de chino, que utiliza el mismo tipo de tubo complejo, es realizada en madera, lo cual nos permite contrastarla con los instrumentos arqueológicos, resaltando la enorme dificultad constructiva que presentan estos últimos. En efecto, la elección de piedra para construir flautas va en contra de todo principio práctico: es quizá el material más difícil de trabajar para obtener un instrumento de viento, habiendo otros mucho más sencillos de trabajar, como la madera, caña o cerámica, cuyos resultados acústicos son frecuentemente similares.

\section{UN ESPÉCIMEN EXCEPCIONAL}

Conocemos una gran cantidad de antaras Aconcagua, entre las cuales destacan unas de porte muy esbelto, con forma cuadrangular bastante orgánica y sumamente fina en su confección. De este tipo de flautas conocemos dos enteras, en excelente estado de conservación (MCHAP-MAS 0073 y MNHN 3516), extraordinariamente parecidas entre sí, a tal punto que podrían haber sido hechas por el mismo artesano. Sin embargo, la primera fue excavada por Berdichewsy en el Cementerio de Bellavista (San Felipe, rio Aconcagua alto) y la segunda ingresada al Museo en 1883 como de «Collipulli», localidad situada en plena región de Araucanía, por lo que pensamos que, o bien se trata de un error, o de un préstamo cultural.

El contexto en que fue descubierta la antara MCHAP-MAS 0073 nos entrega datos importantes: fue encontrada en la tumba con el túmulo más grande del cementerio de Bellavista, donde yacía el único individuo enterrado boca abajo; todos los demás fueron enterrados boca arriba, en tumbas menores, sin instrumentos musicales. Además, se le había depositado la flauta sobre la cabeza, es decir, en un lugar destacado. Todo esto sugiere que el instrumento y su sonido rajado estaban reservados para personajes importantes. La antara parece haber sido parcialmente «matada», ya que un tubo presenta en su parte distal un orificio intencional que lo silencia según se aprecia en la Figura 2. Hemos elegido este instrumento para realizar un estudio acústico más detallado por lo excepcional de su factura, por su estado de conservación, por los datos contextuales que posee y por la disponibilidad a ser manipulada. 


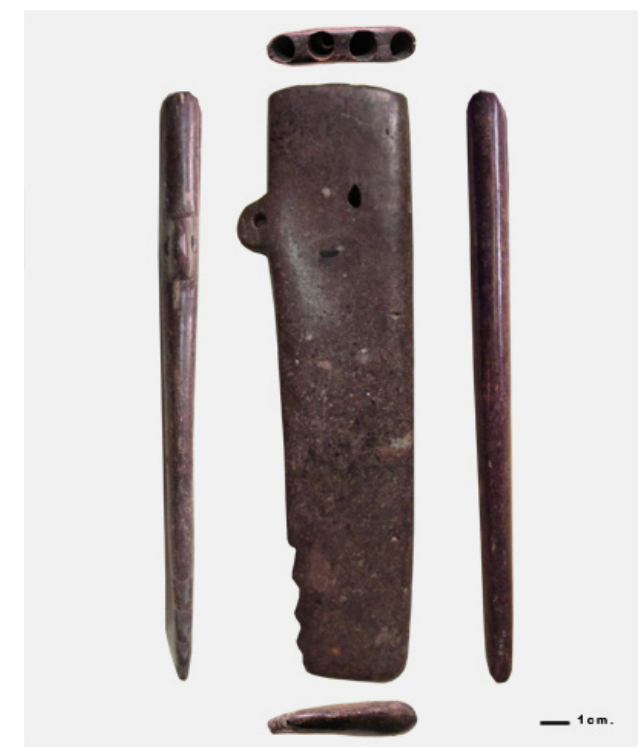

Figura 2. Antara MCHAP-MAS 0073 en sus cuatro vistas. El segundo tubo de izquierda a derecha parece haber sido silenciado.

\section{EL SONIDO RAJADO}

En la descripción física tradicional del sonido (Fletcher 1999) se identifican cuatro parámetros independientes: altura, timbre, intensidad y duración. La música occidental de origen europeo privilegia jerárquicamente la altura, casi a niveles obsesivos, por sobre los otros parámetros, centrando la discusión en la distribución de la coma pitagórica, para finalmente adoptar un sistema temperado adecuado para las manipulaciones de alturas que exigía su evolución estética.

La escena sonora americana prehispánica, en cambio, parece haber optado por una jerarquía diferente, en donde el timbre junto a la intensidad adquieren un sitio privilegiado. Ejemplos de esta elección son el sonido «tara», propio de instrumentos como las tarkas y sikus, y el sonido rajado.

Desde la perspectiva acústica, el sonido rajado se caracteriza por tener una estructura timbrística rica en armónicos, una gran intensidad y una inestabilidad temporal que puede ser percibida como vibrato o frullatto, altamente valorado por los intérpretes de los bailes chinos actuales, quienes la llaman con distintos nombres como gorgoreo, garganteo, ganseo, catarreo o llorona, según su cualidad (Pérez de Arce 2002). Produce una percepción de altura global poco definida o ambigua (Pérez de Arce 2000, Wright 1998) y a veces percibida disonante en su timbre.

Si el resonador de un instrumento de viento tiene forma cilíndrica y abierto en ambos extremos, generará sonidos cuyos componentes armónicos principales se encontrarán ubicados en múltiplos enteros de la frecuencia fundamental. Si el resonador es cerrado en un extremo, se generarán armónicos solamente en múltiplos 
impares de la frecuencia fundamental. En ambos casos el sonido generado es llamado armónico. El sonido rajado puede ser producido, en su geometría más simple, por un tubo complejo consistente en dos cilindros de diferente diámetro, acoplados. Su construcción requiere una gran habilidad, sensibilidad auditiva y un conocimiento muy específico que se ha ido trasmitiendo de generación en generación, ya que precisa de un ajuste muy fino de las relaciones entre el largo y el diámetro de ambos tubos. Actualmente es un oficio dominado exclusivamente en madera y por muy pocas personas. También se puede producir en geometrías más complejas como las observadas en algunas antaras de piedra arqueológicas.

La precisión observada en el trabajo de la piedra de la antara MCHAP-MAS 0073 muestra un conocimiento y un nivel artesanal muy superior al de la "pifilka» actual, habilidad que se prueba dominada a la perfección al ser capaz de producir cuatro tubos complejos en un trozo macizo de piedra. Notable resulta también el hecho de que se encuentren los cuatro tubos cerrados en el extremo distal, lo que evidencia la pericia del constructor para excavar con precisión la piedra para lograr los largos y anchos precisos. En la región diaguita (norte chico), las antaras tienen este extremo abierto, que creemos se cerraba a través de un tapón de madera que permitía realizar un ajuste fino de la relación de largos de los dos tubos para obtener el sonido buscado, lo cual facilita su construcción.

Creemos que la perforación de los tubos se hacía en dos etapas: en una primera, se perforaba

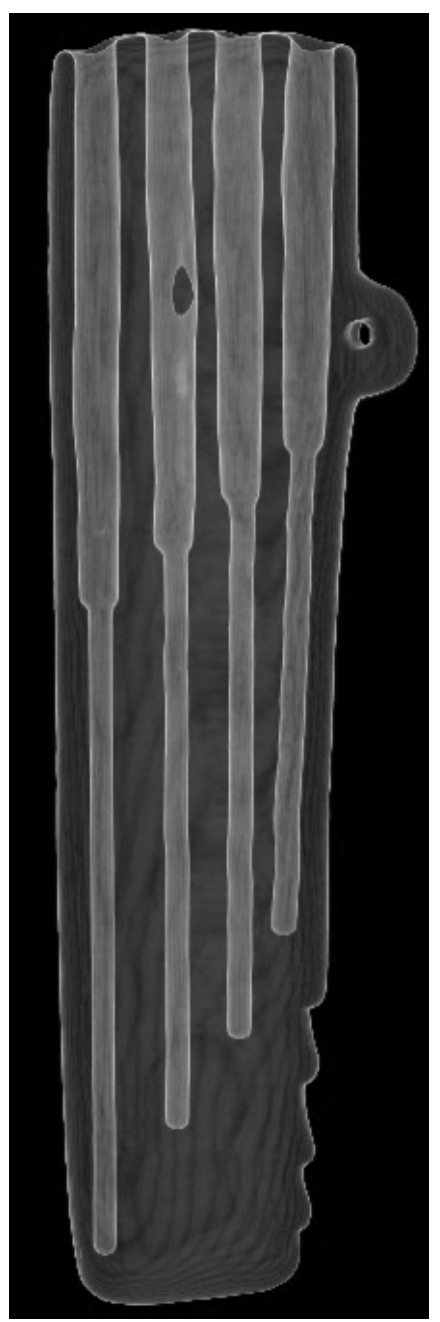

Figura 3. Antara MCHAP-MAS 0073. Vista a través de un scanner. Se observa el detalle del perfil interno de los tubos. con una broca dura (roca dura, cristal o metal ${ }^{1}$ ) en un eje rotativo (de caña o madera) que arrastra el abrasivo. El resultado es un tubo perfectamente cilíndrico, con estrías horizontales muy pequeñas. En las «antaras», por lo general todos los tubos comparten el mismo tipo de diámetro y de perfil interno, lo cual sugiere que se usó una misma herramienta para perforar toda la serie. Luego se

1 No se ha conservado su evidencia en la región. Los mapuches conservan nombres para esta herramienta: pincubue (el punzón), pincún (taladrar, punzonear, agugerear), nombres que guardan estrecha relación con nombres de flautas locales de piedra (ver Pérez de Arce 2008). 
procedía a ensanchar la mitad proximal, mediante un raspado longitudinal que deja, por lo general, estrías marcadas².

Debido a las dimensiones del instrumento, se requiere que la perforación tenga una dirección muy exacta para que no perfore los costados del bloque, ni rompa las paredes entre un tubo y otro. El alineamiento es milimétrico, lo cual le permite mantenerse dentro de el trozo de piedra que es apenas más grueso que el diámetro mayor del tubo. El margen de error observado en el perforado, tanto en los fragmentos que hemos estudiado como usando radiografía (ver Figura 3), alcanza el límite de resistencia del material (hasta $0,7 \mathrm{~mm}$.). La exactitud alcanzada solo se puede explicar por el uso de alguna guía mecánica que evite el desvío de la broca en rangos milimétricos. No conocemos equipos de ese tipo entre las culturas precolombinas, lo cual deja abierta una gran interrogante al respecto.

En resumen, nos hallamos ante una artesanía notablemente desarrollada, que utiliza una materia prima muy específica, la piedra combarbalita rojiza importada desde las canteras de la cordillera de Combarbalá, a ciento ochenta kilómetros de distancia. Esta artesanía está al servicio de una acústica muy sofisticada y alcanza un alarde de virtuosismo que llega hasta el límite mismo de las posibilidades que permite el material, pero que no se exhibe, manteniéndose oculto a la vista. Esos artesanos tenían un nivel de excelencia superior, lo cual nos revela una faceta profunda del quehacer cultural de la época. A la supuesta pobreza las industrias líticas ${ }^{3}$ se oponen la refinadísima técnica referida a estos instrumentos, hoy impensable sin la ayuda de la tecnología.

\section{ELEMENTOS ACÚSTICOS}

El tubo complejo puede aproximarse a través de dos cilindros de distinto diámetro acoplados como se muestra en la Figura 3.

2 Es interesante dejar constancia que este doble método de perforación, uno rotatorio que deja una superficie bastante lisa y otro longitudinal que deja estrías marcadas puede tener un origen local en las «pifilkas acodadas», especie organológia quizá más antigua, y en las cuales la sección ancha presenta estrías sumamente marcadas, a modo de surcos profundos. Esta especie organológica no está bien estudiada, por lo cual no podemos adelantar más en esta hipótesis.

3 La artesanía lítica Aconcagua consiste en percutores, instrumentos cortantes, lascas, raspadores, palas, morteros. elementos de molienda, puntas de proyectil, pulidores de cerámica y adornos, pesas para redes y «chopes», ver Cornejo / Galarce 2000: 113, 114; Sanchez /Massone 1995:22, 24. 


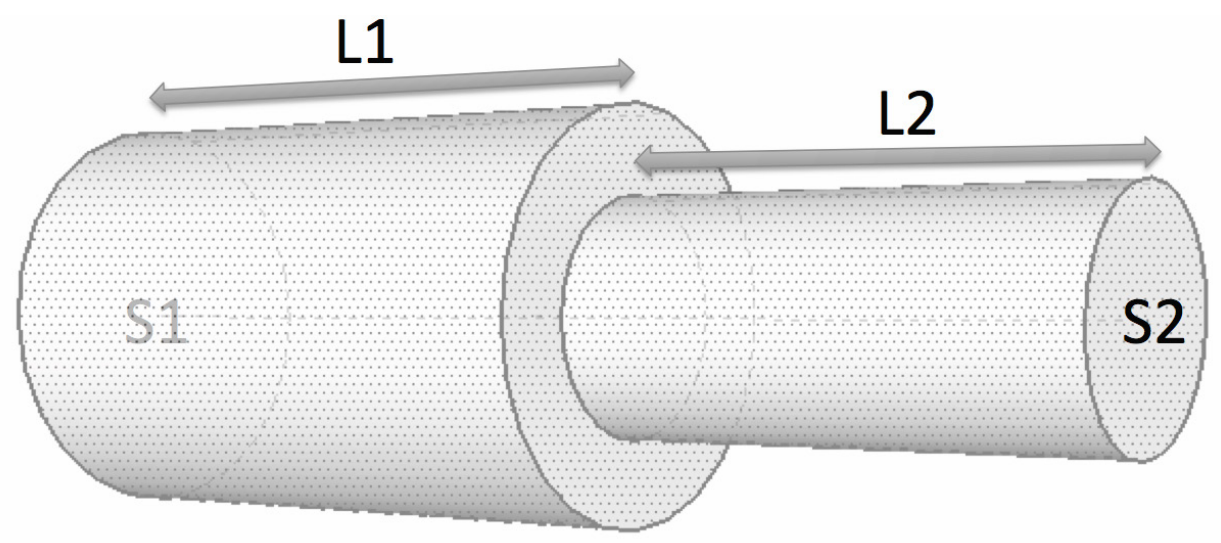

Figura 4: Esquema del perfil interno de un tubo complejo.

Durante el perforado del tubo, la geometría interna debe tratarse con extraordinaria precisión para obtener el sonido rajado. En particular se debe atender a la exacta relación largo-ancho de las dos secciones del tubo, el control de sus diámetros, largos y proporciones internas. En efecto, al modelar acústicamente el resonador complejo como dos cilindros de largo diferente, $L_{1} y L_{2}$, observamos que las frecuencias de resonancia quedan determinadas por la siguiente relación:

$$
\tan k L_{1} \times \tan k L_{2}=\frac{s_{1}}{s_{2}} ; \quad k=\frac{2 \pi f}{c}
$$

donde $c$ es la velocidad del sonido, $\mathrm{f}$ la frecuencia de oscilación, $L_{1} y L_{2}$ son los largos de los cilindros ancho y delgado respectivamente y $S_{1}$ y $S_{2}$ el área de sus secciones.

Para evidenciar la precisión requerida para posicionar los armónicos en un lugar preciso observemos el caso en que ambos largos son iguales, es decir $L_{1}=L_{2}=L$ :

$$
\tan ^{2} k L=\frac{s_{1}}{s_{2}}
$$

que se puede analizar gráficamente buscando la intersección de la curva desplegada (en azul), que representa la $\tan ^{2} k L / \pi$ y una línea horizontal, que representa la razón entre $\frac{s_{1}}{s_{2}}$ : 


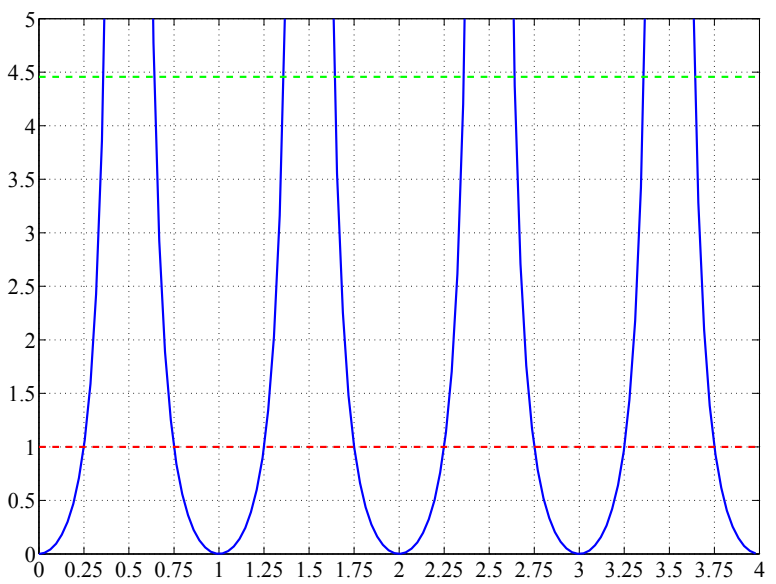

Podemos verificar los casos triviales, por ejemplo, cuando los dos cilindros tienen igual superficie: $\frac{s_{1}}{s_{2}}=1$. Estamos en el caso de un cilindro cerrado de largo $2 L$, con sus armónicos ubicados en múltiplos impares de su frecuencia fundamental, es decir en $k L=0.25 \pi, 0.75 \pi, 1.25 \pi$, etc. Situación que también se verifica asintóticamente cuando la superficie del tubo pequeño es 0 , es decir la relación $\frac{s_{1}}{s_{2}}$ tiende a infinito, tenemos un cilindro cerrado de largo $L$ con frecuencias de resonancia en $k L=0.5 \pi, \pi, 1.5 \pi$, etc.

En el caso de las flautas de sonido rajado, hemos observado que la relación es cercana a 3 , generando un espaciamiento armónico quasi-regular, con frecuencias de resonancia que siguen un doble patrón armónico en series $k_{n} l=\frac{1}{3} \pi+n \pi y \frac{2}{3} \pi+n \pi$. Dicho de otra forma, se produce una serie armónica incompleta en donde se repite el siguiente patrón $k l=\frac{1}{3} \pi, \frac{2}{3} \pi, \frac{4}{3} \pi, \frac{5}{3} \pi, \frac{7}{3} \pi, \frac{8}{3} \pi$, etc, es decir, uno de cada tres armónicos no existe. El tañido del instrumento, junto a su geometría y su disposición de armónicos, propicia una ejecución a gran intensidad generando no linealidades que crean armónicos en la serie completa, incluso en aquellos lugares en donde el resonador complejo las evita.

Si esa relación de superficies no se cumple $\left(\frac{s_{1}}{s_{2}} \approx 3\right)$ se produce un desplazamiento de armónicos irregular hacia frecuencias más agudas y más graves que la serie recién descrita, lo que inhibe la producción del sonido rajado. De manera que la menor imperfección interna del tubo puede hacer fracasar el instrumento.

En las «antaras estudiadas», los largos de los dos tubos son ligeramente diferentes. Adicionalmente, la presencia de los labios del ejecutor en el extremo abierto del instrumento es interpretado acústicamente como una impedancia adicional que extiende el largo acústico efectivo del tubo más ancho. Esta diferencia de longitudes produce que en lugar de una curva regular como la $\tan ^{2} \mathrm{kl}$ nos encontremos con una curva más caprichosa, $\tan k L_{1} \times \tan k L_{2}$, que posiciona algunos parciales cerca de sus posiciones armónicas, pero suficientemente distantes para producir intermodulación que creemos responsable del ganseo propio del sonido rajado. De manera que el delicado balance entre la relación de superficies y largos permite 
que el instrumento produzca el poderoso sonido vibrante, o bien, por un mínimo desajuste, el instrumento sea completamente inservible.

El material de la parte interior del tubo de la flauta puede incidir en su sonido a través de dos mecanismos: por un lado el desplazamiento mecánico de las paredes internas, aunque en la mayoría de las flautas es despreciable ya que el desplazamiento longitudinal de partículas domina al interior del tubo y determina su sonido. En el caso de las «antaras", la onda estacionaria es de tal magnitud que podría inducir un movimiento de las paredes que afecte el sonido producido. Por otro, las pérdidas por la porosidad de las paredes internas del tubo que, al ser de piedra porosas e irregulares $^{4}$, actúan oponiéndose al desplazamiento del frente de ondas y produciendo fricción por la viscosidad del aire, lo que se traduce en pérdidas energéticas disipadas en forma de calor.

La acústica de este tubo responde a una técnica muy precisa de tañido ${ }^{5} \mathrm{y}$ da forma a una estética cultural muy precisa, que si bien desconocemos cómo era hace 500 años, tenemos un referente directo en los bailes chinos, quienes continúan usando "pifilkas» en una música que no ha integrado ningún elemento foráneo a su estética surandina ancestral. El estilo de tocar "pifilka» se extiende con diferencias regionales desde Copiapó hasta Temuco, aproximadamente.

Las «pifilkas» del Aconcagua, llamadas flautas de chino, se caracterizan porque se tañen con un soplido muy vigoroso, que hemos medido en laboratorio verificando que alcanzan presiones en la boca del orden de 4 veces superior a las observadas normalmente en flautas traversas, generando chorros de aire que viajan $75 \mathrm{~m} / \mathrm{s}$, los cuales al salir por la sección de los labios de superficie aproximada de $0.8 \mathrm{~cm}^{2}$ produce un gasto de aire de 6 lts/seg, generando una excitación altamente turbulenta capaz de crear la gran cantidad de parciales observados en el sonido rajado. La posición de los labios en el extremo abierto del resonador aporta una impedancia acústica que puede ser modelada como un tubo adicional conectado a la flauta, de manera que mientras más cubra mayor será su largo. Como hemos visto, la relación entre los dos largos es de vital importancia, de manera que la posición adecuada de los labios combinada con la vigorosa velocidad del chorro de aire direccionado al lugar preciso de la embocadura, permite lograr el correcto equilibrio entre armónicos agudos y graves, y la cualidad vibrada y altamente disonante propia del sonido rajado. Al no cumplirse alguna de estas condiciones, el resonador se pone a vibrar preferentemente

$4 \quad$ La irregularidad y porosidad de la piedra combarbalita es considerablemente mayor al de los metales modernos, a pesar de ser comúnmente considerada como una piedra «lisa». Probablemente en los tiempos que se hizo esta antara esa piedra estaba entre los materiales menos porosos capaces de ser trabajados. Por otra parte la irregularidad de las paredes interiores está, en el caso especifico del ejemplar elegido, y en general en las otras «antaras», reducido al mínimo, con una clara intención de producir paredes muy lisas y de la mayor regularidad interna posible.

5 El instrumento musical responde a una técnica para hacerlo sonar, propia de su cultura: para entender una flauta arqueológica Aconcagua debemos basarnos en la relación entre ella, los datos inferidos de cronistas y viajeros y los datos extrapolados de la etnografía, o de nuestra experiencia. 
en una frecuencia particular produciendo el llamado «sonido botella» 6 . Parte de la técnica del tañido consiste entonces en coordinar la velocidad del chorro de aire con su dirección a la vez que se restringe su forma a través del control fino de la apertura de los labios y la cobertura del extremo abierto del tubo.

Hemos observado también que la acumulación de aire al interior del tubo cerrado eleva la presión estática interior, generando un flujo hacia el exterior que cruza con el chorro de aire ayudando a que se deflecte hacia el canto filoso de la embocadura y facilitando la emisión de sonido.

Humedecer las paredes internas del instrumento es un requisito para obtener su sonido rajado. La amplitud de las crestas de impedancia se ve afectada por la porosidad de los muros internos del resonador, debido al incremento de las pérdidas visco-termales que experimentan las ondas de presión al propagarse al interior del resonador. Es por eso que las pifilkas son humedecidas antes de ser tocadas y posiblemente también lo eran las «antaras» arqueológicas.

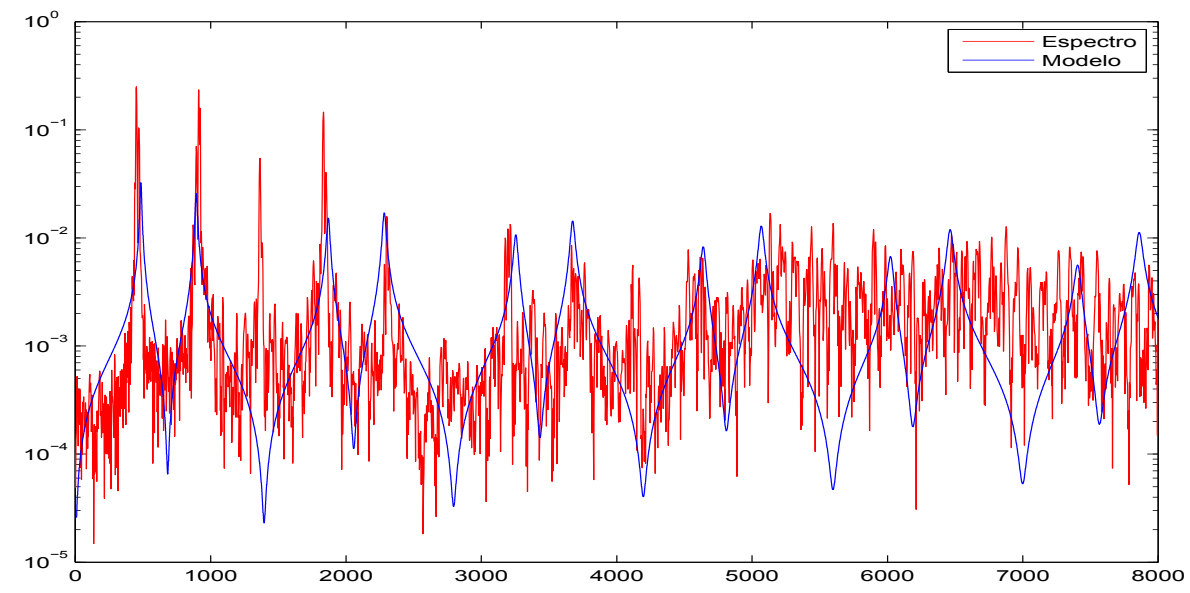

Figura 5. Análisis espectral del sonido irradiado de un tubo de la antara MCHAP-MAS 0073. En azul se observa el modelo de admitancia y en rojo el espectro del sonido irradiado en donde se observa la riqueza de armónicos del sonido rajado.

Solicitamos a Guillermo Díaz, experimentado chino puntero del baile de Pucalán, ejecutar la antara MCHAP-MAS 0073. Logró obtener el sonido rajado en los cuatro tubos, confirmando la excepcionalidad del instrumento. Hemos por lo tanto constatado una relación directa entre el uso musical (sonoro) actual de la flauta de chino y el del pasado arqueológico en la región del Aconcagua y valles vecinos ${ }^{7}$.

6 El «sonido botella» es considerado normalmente un sonido deficiente, un error, salvo en ocasiones muy específicas, por los bailes chinos.

7 Experimentos similares hechos por Claudio Mercado, otro experimentado "chino», en numerosas «antaras» y «pifilkas» aconcagua, diaguita y mapuche confirman esta hipótesis, encontrando incluso correspondencia con los tipos mas preciados de "sonido rajado», actualmente presente en muy pocos ejemplares, las flautas llamadas «catarras». 


\section{SIMULANDO UN RESONADOR COMPLEJO}

Habiendo descrito acústicamente el instrumento, resulta atractivo generar una representación digital, o simulación, de sus características física y como consecuencia de su sonido, con la doble finalidad de restituir el sonido de otras «antaras» que no puedan ser tocadas y contar con una representación digital del sistema acústico que nos permita manipular libremente sus parámetros para comprender con mayor precisión su funcionamiento y obtener nuevos sonidos.

Para simular el comportamiento de un tubo complejo, usamos la técnica de guías de onda ${ }^{8}$ (Smith 1992), en donde la propagación al interior de cada uno de los cilindros emula la solución de d'Alembert de la ecuación de onda en una dimensión (Kinsler 2000, Chigne 2008) y puede ser implementada a través de una línea de retardos digitales como se muestra en la Figura 5. También se deben considerar filtros que den cuenta de las pérdidas visco-termales al interior del tubo y de la reflexión de la onda en su extremo abierto.

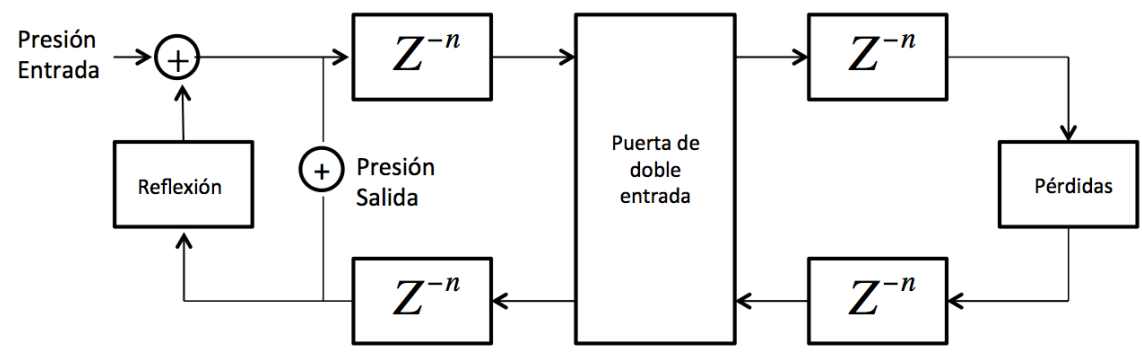

Figura 6. Esquema de la simulación computacional de un resonador complejo.

El descanso o la unión de los dos tubos puede ser simulada a través de una juntura de doble puerta, que da cuenta de la reflexión parcial y la propagación acústica que ocurre en la unión (Valimaki 1995).

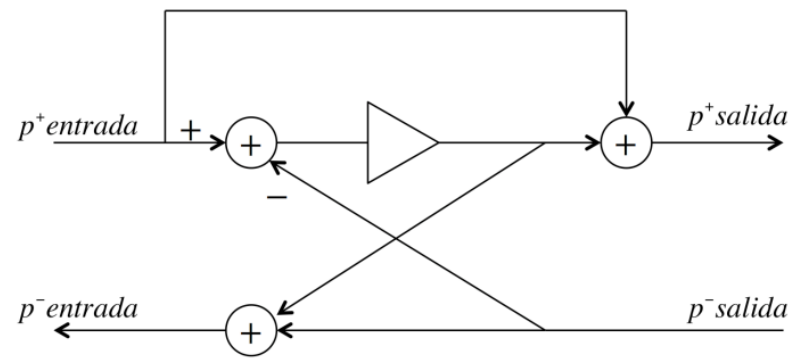

Figura 7. Esquema de la implementación eficiente de la juntura de doble puerta que simula computacionalmente el «descanso» del instrumento (Valimaki 1995).

Del inglés waveguides. 
Este sistema, implementado digitalmente en un computador y estimulado con una excitación apropiada, es capaz de producir sonidos de manera similar al tubo de la antara, como lo muestra la comparación de la Figura 7, donde se observa la impedancia de entrada de un tubo modelado analíticamente y simulado. Observamos que las frecuencias de resonancia coinciden aunque presentan una diferencia en amplitud que puede ser compensada con un ajuste fino de los filtros de reflexión y pérdidas.

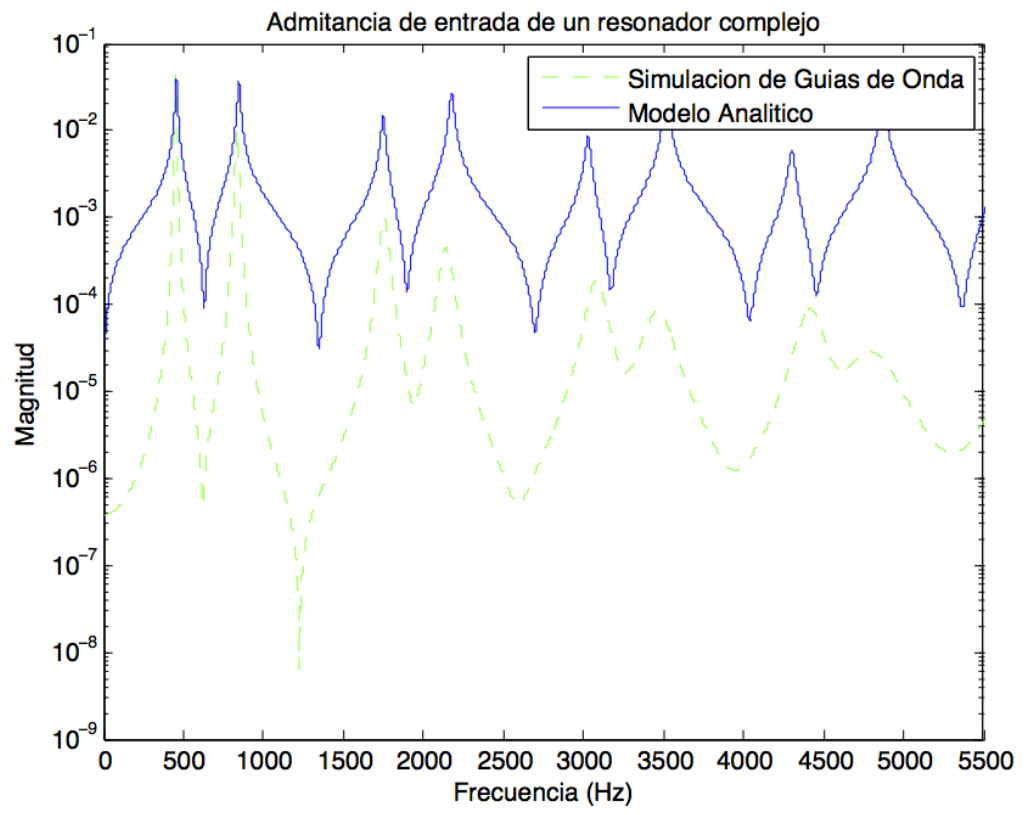

Figura 8. Admitancia de entrada de un tubo complejo. Modelado analíticamente (azul) y simulado con guias de onda (verde).

\section{CONCLUSIONES}

Las «antaras surandinas» prehispánicas comparten una misma tipología organológica, son de piedra, tienen 4 tubos (raramente 3 o 2) capaces de dar el sonido rajado, cavados en disposición escalerada. Presentan una enorme difusión temporal y espacial que, ciertamente, no se debe a la facilidad de construcción, ni tampoco a una permanencia estilística externa. La explicación está en el terreno acústico, en donde observamos una tecnología suficientemente interesante como para haberse difundido y perdurado inclusive al ser traspasado de un tipo de instrumento a otro a través del tiempo y la distancia. Hemos intentado aquí develar algunos de los principios acústicos escondidos tras esta tecnología.

Desde su lejano origen durante la expansión Tiwanaku en el desierto de Atacama hasta Chile Central, a miles de kilómetros y a cientos de años de distancia, la «antara» destaca como el instrumento ritual más importante dentro del orden social y espiritual. 
Podemos suponer que en todos los contextos, la «antara», con sus rangos disonantes de sonido propicios para la obtención de otros estados de conciencia, se posiciona por encima de otros instrumentos musicales (trompetas, tambores, quenas, silbatos, sonajas, etc.), en términos de estructura y organización musical. El sonido rajado, más intenso que el de las quenas, trompetas, tambores y sonajeros, tenía que haber sobresalido durante las fiestas rituales, donde la competencia de volumen sonoro entre bailes es de gran importancia ${ }^{9}$. La permanencia de objetivos acústicos mantenida en el tiempo a través de diferentes lugares implica una comunicación social entre artesanos y músicos, junto a un conocimiento profundo de los usos y funciones de ese sonido. Este sistema estético alcanza en el Complejo Cultural Aconcagua su máxima expresión, tal vez como consecuencia indirecta de la importancia local que tuvo, y sigue teniendo aún, la competencia sonora durante los rituales. Su factura es más compleja, más fina, delicada y precisa que los otros instrumentos. Su relación con la parafernalia destinada a la ingesta ritual de plantas psicoactivas concuerda con el uso generalizado de la música en el contexto de prácticas chamánicas en América.

No sabemos cuánto ha cambiado la música desde que desapareció la antara hace quinientos años o más, pero reconocemos su impronta en la música de flautas de los actuales bailes chinos que tocan «pifilkas» pareadas de sonido complejo y disonante, extendido armónicamente en conjuntos e interpretadas en forma puramente instrumental. Esta forma de hacer música es muy característica de la cultura andina y persigue borrar la diferencia entre el individuo y el conjunto ${ }^{10}$, sonando todos como un gigantesco instrumento que representa a una comunidad, una imagen sónica que identifica al grupo, la cual durante las grandes fiestas rituales entra en competencia con otros grupos similares, generando una característica polifonía multiorquestal. Los instrumentos arqueológicos y los actuales son acústicamente semejantes. Los primeros muestran mayor perfección de formas y de materiales, y su manufactura requería de mayor tiempo y esfuerzo. Esto se explica por haber pertenecido a una cultura viva que cuidaba su más preciada expresión musical dedicándole tiempo y sabiduría, en contraste con los bailes actuales que están luchando hace siglos contra una sociedad que los ha querido aniquilar. Por otra parte, el sistema ritual en que se inscribe sonido rajado esta el diseñado para producir un estado de trance en el músico, lo cual permite una integración entre cuerpo, mente y espíritu que disuelve las parcialidades, y lo pone en contacto con las potencias de la naturaleza que rigen los destinos de su comunidad.

La «antara» fue en la cultura Aconcagua el instrumento ritual más importante y, consecuentemente, el objeto artesanal más elaborado y refinado, alcanzando niveles de excelencia extraordinarios. Su status estaba avalado por una herencia larga y poderosa en que su sonido, su cualidad y potencia acompañaba el sacrificio

9 Existen esquemas específicos relativos a esta competencia sonora, cuyo resultado es una polifonía multiorquestal, ver Pérez de Arce 1997.

10 En Conima, Sur del Perú, se habla de «sonar como un solo instrumento». Ver Turino 1988:75; Gérard 2009:125. 
humano por decapitación, máximo exponente del ritual andino, ritual potenciado por la obtención de otros estados de conciencia. El conocimiento de estas estructuras acústicas que permiten el sonido rajado perduró, pasando por generaciones, hasta los bailes chinos actuales, soportando cambios radicales en la ritualidad y la cultura, prohibiciones y acoso por parte de la iglesia y de la sociedad urbana, en una presión sostenida durante medio siglo en la zona más poblada y occidentalizada de Chile.

\section{REFERENCIAS}

Ampuero, Gonzalo. «Cultura Diaguita». Serie Patrimonio cultural Chileno. Santiago: Ministerio de Educacion, 1978. Medio impreso.

Ampuero, Gonzalo. Antiguas culturas del Norte Chileno. Santiago: Museo Chileno de Arte Precolombino, 1986.15-29. Medio impreso.

Berenguer, José. Caravanas, interacción y cambio en el Desierto de Atacama. Santiago: Sorawi / Museo Chileno de Arte Precolombino, 2004. Medio impreso.

Castillo, Gastón 1992 «Evidencias sobre uso de narcóticos en el norte semiárido chileno Catastro regional». Boletín del Museo Regional de Atacama 4 (1992). 105- 160. Medio impreso.

Chaigne, Antoine y Jean Kergomard. Acoustique des instruments de musique. París: Belin, 2008. Medio impreso.

Cornejo, Luis y Patricio Galarce. «Avances en el Estudio de la Litica de Sociedades Tardías de Chile». XV Congreso Nacional de Arqueologia Chilena (2000). Medio impreso.

Ebert, James. An Ethnoarcheological Approach to Reasessing the Meaning of Variability in Stone Tool Assemblages, Implications of Ethnography for Archaeology. Ed. Carol Kramer. Nueva York: Columbia University Press, 1979. 59 - 74. Medio impreso.

Falabella, Fernanda, Lorena Sanhueza, y Eugenia Fonseca. «Las materias primas de la cerámica aconcagua salmón y sus implicancias para la interpretación de la organización de la producción alfarera». Chungará 34, 2 (2002). 167-189. <http:// www.scielo.cl/scielo.php?script=sci_arttext\&pid=S0717>. Sitio web.

Fletcher, Neville y Thomas D. Rossing. The Physics of Musical Instruments. Nueva York: Springer, 1999. Medio impreso.

Gérard, Arnaud. «Sonidos ondulantes» en silbatos dobles arqueológicos ¿una estética ancestral reiterativa?» Revista española de Antropología Americana 39, 1 (2009) 125-144. Medio impreso.

---. Tara y tarka. «Un sonido, un instrumento y dos causas (estudio organológico y acústico de la tarka)». Diablos tentadores y pinkillus embriagadores. La Paz: Plural, 2009. 69-149. Medio impreso.

Helmholtz, Hermann. On the Sensations of Tone as a Physiological Basis for the Theory of Music. 1886. Nueva York: Dover Publications, 1954. Medio impreso. 
Hidalgo, Jorge. «Diaguitas Chilenos Protohistóricos». Culturas de Chile, Prehistoria, desde sus orígenes hasta los albores de la conquista. Santiago: Andrés Bello, 1989. Medio impreso.

Horta, Helena. «Sacrificadores y Victimas. Hacia la definicion de un estilo circumpuneño en el complejo alucinógeno (zona atacameña y noroeste argentino)». The Southern Andean Iconographic Tradition. Dumbarton Oaks / Cotsen Institute of Archaeology / Universidad de Chile / State University of New York, 2010.

Kinsler, Lawrence, Austin Frey, Alan Coopens, y James V. Sanders. Fundamentals of Acoustics. Nueva York: John Wiley \& Sons, 2000. Medio impreso.

Latcham, Ricardo «La organización social y las creencias religiosas de los antiguos araucanos». Publicaciones del Museo de Etnologia y Antropología de Chile 2,3,4 (1922). Medio impreso.

Lawson, Graeme. «Music, Intentionality and Tradition: Identifying Purpose, and Continuity of Purpose, in the Musicarchaeological Record, Vorträge des 3». Symposiums der Internationalen Studiengruppe Musikarchäologie in Kloster Michaelstein 9-16 (2002). 61 -97. Medio impreso.

Niemeyer, Hans. "Pasos cordilleranos y contactos entre los pueblos del norte chico de chile y el noroeste argentino». La Cordillera de los Andes: ruta de encuentros. Santiago: Museo Chileno de Arte Precolombino, 1994. 23-37. Medio impreso.

Nuñez, Lautaro. "Problemas en torno a la tableta de rape». Anales de la Universidad de Antofagasta (1963). Medio impreso.

Pérez de Arce, José. «Sonido Rajado, the Sacred Sound of Pifilcas». Galpin Society Journal (1997). 17-50. Medio impreso.

---. «Sonido Rajado, historical approach». Galpin Society Journal (2000) 233 - 251. Medio impreso.

---. «Etnographic Analogies Between Southandean Patterns of Dance-Music Traditions and it Prehispanic Evidence». 3rd Symposium of the International Study Group on Music Archaeology, Foundation (2002). Medio impreso.

---. «Influencia musical de Tiwanaku en el Norte de Chile: el caso del «siku» y de la «antara»". Tiwanaku, Aproximaciones a sus contextos históricos y sociales. Comp. Mario A. Rivera y Alan L. Kolata. Santiago: Universidad Bolivariana, 2004. 193 - 220. Medio impreso.

---. «The Shape of Trance in Central Chile and Araucania». XII. Congress of the ICTM Study Group for Music Archaeology. Universidad de Vayadolid. 20-22 de septiembre del 2011. Presentación en congreso.

---. «Diseño del Trance». XII Congress of the ICTM Study Group for Music Archeology, Universidad de Valladolid. España, 2012. Presentación en congreso.

Smith, Julius O. «Physical Modeling using Digital Waveguides». Computer Music Journal 16, 4 (1992). 74-91. http://www.jstor.org/stable/3680470. Sitio web.

Sanchez, Rodrigo y Mauricio Massone. Cultura Aconcagua. Santiago: Dirección de Bibliotecas, Archivos y Museos, 1995. Medio impreso. 
Torres Manuel Constantino et al. «Snuff Powders from Prehispanic San Pedro de Atacama: Chemical and Contextual Analysis». Current Anthropology 32, 5 (1991). 640-649. Medio impreso.

Valimaki, V. «Discrete-time modeling of acoustic tubes using fractional delay filters,» University of Technology, Finland, 1995. Tesis de doctorado.

Wright, H. A. K. y D. M. Campbell. "Analysis of the Sound of Chilean Pifilca Flutes». The Galpin Society Journal 51 (1998). Medio impreso. 